Nelson Pinheiro Gomes, Clarissa Martins Alves Lopes, William Afonso Cantú, Gilbertto Prado *

\title{
Análise Estratégica de Tendências Socioculturais: uma triangulação de métodos científicos
}

Nelson Pinheiro Gomes é Professor Auxiliar do Programa em Cultura e Comunicação da Faculdade de Letras da Universidade de Lisboa. Doutorado na Especialidade de Cultura e Comunicação; desenvolve investigação em Estudos de Cultura (Estudos de Tendências e Gestão da Cultura) no CEAUL - Centro de Estudos Anglísticos da Universidade de Lisboa. <nelsonpinheiro@campus.ul.pt> ORCID: 0000-0003-3724-4044
Resumo 0 principal objetivo deste artigo passa por ilustrar os potenciais benefícios na articulação de métodos e práticas como a semiótica, a análise de conteúdo e a imersão urbana no contexto dos Estudos de Tendências. Desta forma, articulando os principais autores que trabalham a análise de tendências socioculturais e estas práticas metodológicas, é possível rever o processo de recolha, sistematização e análise da informação, conferindo um maior rigor científico ao processo de identificação e interpretação de tendências. Pretende-se, assim, contribuir para um maior mapeamento de métodos que possam ser úteis aos Estudos de Tendências, bem como promover uma contínua revisão dos processos analíticos associados à interpretação de grandes padrões de comportamento e mudanças nas mentalidades.

Palavras chave Estudos de Tendências, Tendências Socioculturais, Métodos Qualitativos, Triangulação Cultural. 


\section{Strategic Analysis of Sociocultural Trends: a triangulation of scientific methodss}

Clarissa Martins Alves Lopes é Docente de Pós-Graduação no Centro Universitário Belas Artes de São Paulo e na Unochapecó e Docente de Graduação na Faculdade Energia. Doutora em Design pela Universidade Anhembi-Morumbi com período sanduíche no Programa de Cultura e Comunicação da Faculdade de Letras da Universidade de Lisboa (FLUL) ${ }^{1}$. Atua como consultora nas áreas de tendências, inovação e design. <clarissamartinsalves@gmail.com> ORCID: 0000-0002-3456-2162
Abstract The main objective of this paper is to illustrate the potential benefits of combining methods and practices such as semiotics, content analysis and urban immersion in the context of Trend Studies. By articulating the main authors working on the analysis of socio-cultural trends and these methodological practices, it is possible to review the process of collecting, systematizing and analyzing information. This gives greater scientific rigor to the process of identifying and interpreting trends. We intend to contribute to a greater mapping of methods that may be useful for Trend Studies. As well as promote a continuous review of the analytical processes associated with the interpretation of large behavior patterns and changes in mindsets.

Keywords Trend Studies, Sociocultural Trends, Qualitative Methods, Cultural Triangulation.

Análisis estratégico de tendencias socioculturales: una triangulación de métodos científicos

William Afonso Cantú é Docente no IPAM/IADE - Universidade Europeia. Doutorando no Programa em Cultura e Comunicação na Faculdade de Letras da Universidade de Lisboa. Desenvolve investigação em Estudos de Cultura (Estudos de Tendências e Análise Cultural) no CEAUL - Centro de Estudos Anglísticos da Universidade de Lisboa. <williamcantu@campus.ul.pt> ORCID: 0000-0003-4079-9884
Resumen El objetivo principal de este artículo es ilustrar los beneficios potenciales de la combinación de métodos y prácticas como la semiótica, el análisis de contenido y la inmersión urbana en el contexto de los estudios de tendencias. De esta manera, articulando los principales autores que trabajan en el análisis de las tendencias socioculturales y estas prácticas metodológicas, es posible revisar el proceso de recolección, sistematización y análisis de información, dando mayor rigor científico al proceso de identificación e interpretación de tendencias. Se pretende, por tanto, contribuir a un mayor mapeo de métodos que puedan ser de utilidad para los Estudios de Tendencias, así como promover una revisión continua de los procesos analíticos asociados a la interpretación de grandes patrones de comportamiento y cambios de mentalidad.

Palabras clave Estudios de Tendencias, Tendencias Socioculturales; Métodos Cualitativos, Triangulación Cultural.
Gilbertto Prado é Professor do Programa de Pós-Graduação em Design da Universidade Anhembi Morumbi e do Programa de Pós-Graduação em Artes Visuais da Universidade de São Paulo. Coordenador do Grupo Poéticas Digitais, trabalha com arte em rede e instalações interativas. É pesquisador $1 \mathrm{~A}$ do CNPq. <gttoprado@gmail.com> ORCID: 0000-0003-2252-3489 


\section{Introdução}

Os Estudos de Tendências são alvo crescente de atenção por parte de investigações científicas individuais, e/ou coordenadas. Académicos e jovens investigadores dedicam-se cada vez mais à revisão do estado da arte sobre esta abordagem, mapeando práticas profissionais e contextualizando as mesmas à luz de contextos científicos. Neste sentido, pretende-se rever os contributos de autores recentes como Rech et al. (2020), Kongsholm e Frederiksen (2018), Rech (2017), Cramer et al. (2016), entre outros. Este autores problematizam conceitos e resumem práticas, considerando contributos anteriores e perspetivas já consolidadas na abordagem como as de Raymond (2010), Higham (2009) e Vejlgaard (2008). Neste âmbito, a problematização de práticas de investigação contidas em autores como Cantú et al. (2019) e Gomes et. al (2018) não só introduzem um mapeamento sobre os cruzamentos entre práticas profissionais e métodos científicos, como apontam para a necessidade deste tipo de trabalhos.

Partindo deste quadro conceptual e metodológico, apresentamos neste artigo um conjunto de possibilidades de articulação entre os objetivos associados aos processos de análise de tendências e diferentes métodos hermenêuticos e de trabalho de campo que podem conferir uma melhor base de replicação processual e contextualização científica dos Estudos de Tendências. Abordamos especificamente o potencial da semiótica, da análise de conteúdo e da práticas contidas na perspectiva de walkscapes de Careri (2013). Importa sublinhar que esta nossa proposta parte do trabalho de revisão e sistematização literária desenvolvida por Cantú et al. (2019), apresentando uma nova leitura sobre as potenciais aplicações metodológicas. Os métodos e práticas aqui descritos apoiam: a observação e recolha de dados, a sua sistematização, e a sua análise e interpretação.

Esta inclusão de métodos reconhecidos, no âmbito das Humanidades e das Ciências Sociais, no contexto da identificação e análise de tendências socioculturais, não só ajuda a estruturar as abordagens analíticas, como permite a replicação dos processos e dos diferentes estudos. Esta questão é muito importante para garantir o desenvolvimento dos Estudos de Tendências no plano profissional, tal como no campo acadêmico.

No final deste artigo, demonstramos a potencial aplicação destes métodos nos exercícios de investigação de tendências, considerando diferentes objetivos, questões e cruzamentos. 


\section{Estudo de Tendências e Cruzamentos Metodológicos}

Muito se tem escrito sobre identificação e análise de tendências socioculturais nos últimos anos (POWERS, 2018, 2019; KONGSHOLM e FREDERIKSEN, 2018; DRAGT, 2017; MASON et al., 2015; RAYMOND, 2010; HIGHAM, 2009; VEJLGAARD, 2008), inclusive em contexto científico e académico (RECH et al., 2020; GOMES et al., 2018; VISONÁ, 2018; CRAMER et al., 2016; RECH, 2017; GOMES, 2017, 2015). Não obstante, ainda há espaço para explorar a articulação de tendências já identificadas com métodos qualitativos e exploratórios ou descritivos que contextualizam a analisam fenómenos socioculturais específicos (RECH, 2020) [1]. Isto traz consigo todo um conjunto de possibilidades estratégicas, mas importa começar por contextualizar o estudo de tendências socioculturais como uma abordagem científica associada aos Estudos de Cultura (GOMES et al., 2018; POWERS, 2019) e com fortes relações históricas às áreas do design, do marketing, entre outras. No campo do design, por exemplo, os Estudos de Tendências consideram a complexidade contextual dos produtos, buscando alertar e informar precocemente profissionais de design a respeito de tendências sociais que possam operar alguma influência sobre aquilo que projetam, além de prover ferramentas intelectuais que permitem a reflexão a respeito dos significados e impactos éticos destas mesmas tendências (MARGOLIN, 2007).

Os Estudos de Tendências visam, primeiro que tudo, identificar e analisar tendências socioculturais, entendendo estas mesmas, num contexto partilhado pela maioria dos autores, como uma direção ou uma mudança (KONGSHOLM e FREDERIKSEN, 2018; DRAGT, 2017; HIGHAM, 2009; VEJLGAARD, 2008; entre outros). Estas mudanças ocorrem ao nível das mentalidades, e de padrões de comportamento associados a práticas e representações (GOMES et al., 2018). As tendências socioculturais habitam o campo das mentalidades (GOMES et al., 2018) e são dinâmicas nas suas transformações ao nível da mente coletiva, ou seja, indicam transformações que ocorrem nas esferas individual, social, cultural e estética (CAMPOS, 2020). Sobre isto, Devon Powers menciona que a cultura floresce através da relação entre indivíduos, comportamentos e objetos que podem simbolizar algo maior no contexto das comunidades (POWERS, 2019). Nessa perspetiva, a autora reflete a respeito da relevância de um estudo aprofundado de todas as 'formas culturais' (manifestações) mediante a observação de um 'modo de vida'", e de forma a entender as tendências tanto numa perspetiva micro ('unidades culturais') quanto numa perspetiva macro (a cultura como um todo) (POWERS, 2019, pp. 33-35). Não obstante, as tendências socioculturais manifestam-se no campo tangível e analisável do quotidiano onde podemos observar e estudar padrões de comportamentos associados a práticas ou a produtos/serviços específicos que se desenvolvem a partir destas mudanças coletivas num processo de difusão documentado e explanado por Rogers (2003), Vejlgaard (2008) e Powers (2019). 
O processo de identificação de tendências tem sido documentado em vários formatos, estando alguns mais relacionados com uma aplicação real (de mercado) (KONGSHOLM e FREDERIKSEN, 2018; DRAGT, 2017; MASON et al., 2015; RAYMOND, 2010) e outros orientados para o desenvolvimento conceptual e técnico desse conhecimento (GOMES et al., 2018; CRAMER et al., 2018; RECH e CAPONERA, 2018).

É comum encontrar propostas de estruturas analíticas que passam por (1) uma fase de observação e recolha de dados onde se usam diferentes práticas para observar produtos, práticas, representações e mais manifestações socioculturais; (2) o processo de sistematização onde os dados e informações recolhidos são sistematizados e categorizados de acordo com afinidades comportamentais e temáticas; (3) uma fase onde as categorias são revistas, articulam-se leituras e são geradas as hipóteses e consequentemente os textos definidores e descritivos das tendências. Mesmo com perspetivas específicas e pequenas nuances processuais, estes três passos são comuns a diferentes autores (GOMES et al., 2018; DRAGT, 2017; CRAMER et al., 2016) e apoiam um processo unificado e aceite pela comunidade para a identificação e análise de tendências. Importa também sublinhar o contributo de RAYMOND (2010) que sublinhou a importância da triangulação cultural - um processo de observação, recolha e estudo de dados através de diferentes métodos com o objetivo de confirmar uma hipótese (PORTA e KEATING, 2008) - numa prática que liga a intuição e a recolha de dados, com uma clara inspiração antropológica e sociológica. A análise desta proposta também pode ser observada em Rech e Nascimento (2017), que reforçam a índole qualitativa e transdisciplinar da 'triangulação cultural'. O mérito da proposta abriu caminho para outros ponderarem a possibilidade que cruzar métodos no âmbito dos Estudos de Tendências. Neste contexto, surge então a questão sobre a natureza científica dos Estudos de Tendências e o seu enquadramento académico. Tendo florescido num contexto empresarial e técnico, a integração do estudo de tendências na academia é um exercício póstumo. A natureza plural ao nível dos conceitos e práticas associados a estes estudos justifica também o seu enquadramento numa não-disciplina (GOMES et al., 2018; INGLIS, 1993), em algo que ultrapassa as balizas comuns das áreas disciplinares. Os Estudos de Cultura, num posicionamento entre as Humanidades e as Ciências Sociais, são um espaço onde os Estudos de Tendências - como abordagem com conceitos e práticas específicos mas que se articulam plenamente com esta área - podem vingar, no seio de diferentes temáticas, missões e objetivos. Contudo, importa ser mais específico sobre esta associação. Não é aqui o espaço para indicar todas, mas não queremos deixar de referir a importância sugerida por Raymond Williams na identificação de padrões e na categorização da cultura como algo social, o todo um modo de vida (WILLIAMS, 1965); bem como, num passo seguinte a esta definição, o entendimento da cultura como práticas significantes que Stuart Hall (1997) sublinhou e que evidencia, aliás, a ligação metodológica dos pontos seguintes ao nível da produção e impressão de significados. 
Entra em cena a Semiótica. Esta tem um papel importante não só na análise de objetos durante o processo de identificação de tendências, mas também na ligação entre tendências identificadas e as demais manifestações socioculturais do quotidiano. Neste segundo plano, ao invés da semiótica americana, partimos da abordagem continental que se alicerça nos pressupostos de Saussure, desenvolvidos por Roland Barthes (1972, 1986) e que estão na base de perspectivas analíticas estratégicas de autores como Gillian Rose (2001) e Laura Oswald (2012). A simplicidade da díade proposta por esta corrente (signo = significado + significante) oferece um quadro analítico capaz de compreender peças de comunicação, tal como outras práticas, representações e artefactos, na medida em que verifica a produção e a impressão de significados nos objetos.

Como Barthes contextualiza, o "significante é um mediador" e ele tem uma substância material ("sons, objetos, imagens") (BARTHES, 1986, p. 47); enquanto o significado é a "representação mental da coisa" (BARTHES, 1986, p. 42), ou seja, a ideia mental que surge através do veículo, do significante. Neste sentido, a ligação entre o significado e o significante dão origem ao signo. Tal como Barthes acrescenta, "o signo é então uma composição de significado e significante. O plano dos significantes é o da expressão e o dos significados é o plano do conteúdo" (BARTHES, 1986, p. 39) ${ }^{3}$.Esta perspetiva permite analisar os significados presentes nos demais objetos de estudo e compreender a separação entre a "coisa" e o seu "sentido".

Mas o contributo mais pertinente surge ao nível das possíveis camada de leitura dos significados e como os mesmos podem ser construídos. Referimo-nos especificamente à questão da denotação e da conotação (BARTHES, 1986). Quando o signo se torna o significado e permite uma outra leitura, então "lidamos com dois sistemas de significação que estão interligados" (BARTHES, 1986, p. 89). Como o autor explica (BARTHES, 1986), no primeiro plano temos o significante e o significado que dão origem ao signo denotativo e no segundo plano este signo dá origem ao um significante que, por sua vez, dá origem a um novo significado: "os significantes da conotação [...] são compostos por signos (significantes e significados unidos) do sistema denotativo" (BARTHES, 1986, 91)4. Os signos denotativos descrevem algo (ROSE, 2001) e representam uma função semelhante à do dicionário, indicando o "conceito como facto" (OSWALD, 2012, p. 54). Os signos conotativos carregam significados com um nível/profundidade maior (ROSE, 2001). Ou seja, enquanto a leitura denotativa permite compreender os significados mais literais e imediatos, a leitura conotativa pressupõe uma segunda ordem de significados mais complexos. Tal como Oswald acrescenta:

The connotative function of discourse endows signs with nuances and shades of meaning that people associate with the brand or any of the brand signifiers and is highly dependent on the context of the message. Connotations may be culturally based, such as the interpretation of sym- 
bols and colors. Connotations may also be highly personal. For instance, some people will see the McDonald's icon and think "clean, predictable place to eat" and others may think "cheap, fattening food" (OSWALD, 2012, pp. 54-55).

Esta leitura conotativa é o que permite o surgimento dos mitos, como Barthes (1972) os entende. O autor indica que o mito é uma segunda ordem do sistema, mas acreditamos que pode ser visto como uma terceira ordem construída em cima do significado, ou dos significados conotativos. Conforme o processo sugerido por Barthes (1972), do plano denotativo surgem os novos significantes para os significados conotativos, algo gerado no seio de uma convenção social. Conforme o autor contextualiza:

\footnotetext{
In myth, we find again the tri-dimensional pattern which I have just described: the signifier, the signified and the sign. But myth is a peculiar system, in that it is constructed from a semiological chain which existed before it: it is a second-order semiological system. That which is a sign (namely the associative total of a concept and an image) in the first system, becomes a mere signifier in the second. We must here recall that the materials of mythical speech (the language itself, photography, painting, posters, rituals, objects, etc.), however different at the start, are reduced to a pure signifying function as soon as they are caught by myth. Myth sees in them only the same raw material; their unity is that they all come down to the status of a mere language (BARTHES, 1972, p. 113).
}

O mito pode funcionar como uma composição de signos denotativos e conotativos. Conforme Barthes (1972) sugere, os significados denotativos dão lugar a uma nova composição ao nível conotativo que se materializa em mitos, sendo que dois dos casos analíticos que o autor trabalhou foram a publicidade e a capa de revistas.

A par da análise semiótica, a análise de conteúdo permite uma leitura sistematizada de séries de dados com maiores dimensões, para além do estudo de caso com objetos muito específicos e em número limitado. Tal como Gillian Rose indica, "a análise de conteúdo estava preocupada com a análise da ideia de quantificação e com uma metodologia das ciências naturais" (ROSE, 2001, p. 54). A autora acrescenta ainda que o método está associado à contabilização da frequência de certos elementos dentro de uma amostra e que o foco está na análise dessas frequências (Rose, 2001). Neste contexto, importa referir o trabalho de Bardin (1979) que mapeou e fundamentou o método, sublinhando nele a "descoberta de conteúdos e de estruturas que confirmam o que se procura demonstrar a propósito das mensagens", ou então "o esclarecimento de elementos de significações sus- 
ceptíveis de conduzir a uma descrição de mecanismos de que a priori não detínhamos a compreensão" (BARDIN, 1979, p.29). Para a autora, a análise de conteúdo pode ter um recorte ao nível dos significados e das temáticas ou dos próprios significantes, com uma função heurística e exploratória e/ ou uma função de verificação de hipóteses (BARDIN, 1979). Assim, no que diz respeito à natureza da análise de conteúdo, Bardin acrescenta:

\footnotetext{
Designa-se sob o termo de análise de conteúdo: Um conjunto de técnicas de análise das comunicações visando obter, por procedimentos, sistemáticas e objectivos de descrição dos conteúdos das mensagens, indicadores (quantitativos ou não) que permitam a inferência de conhecimentos relativos às condições de produção/recepção (variáveis inferidas) destas mensagens. (BARDIN, 1979, p. 42)
}

Esta é uma abordagem rica para a análise de um conjunto de dados/ objetos semelhantes, permitindo descobrir padrões e os significados dessas ocorrências e repetições. Pode ter lugar numa perspetiva diacrónica e sincrónica e que, mediante uma boa categorização, também coloca em contexto objetos singulares. Sobre o processo específico da análise de conteúdo, Wimmer e Dominick apresentam uma súmula coerente e clara do mesmo:

The initial stages of analysis can easily be combined. Nonetheless, the following steps may be used as a rough outline: 1 . Formulate the research question or hypothesis. 2. Define the universe in question. 3. Select an appropriate sample from the population. 4 . Select and define a unit of analysis. 5. Construct the categories of content to be analyzed. 6. Establish a quantification system. 7. Train coders and conduct a pilot study. 8 . Code the content according to established definitions. 9. Analyze the collected data. 10. Draw conclusions and search for indications. (WIMMER e DOMINICK, 2011, p. 160).

Estes métodos permitem leituras hermenêuticas que informam em profundidade tanto largas séries de dados como objetos específicos. Estes objetos e fenômenos encerram em si significados e são a materialização das mentalidades e das diversas mudanças nas dinâmicas socioculturais. Esta desconstrução dos significados impressos e da sua produção ilumina as pequenas e muitas vezes invisíveis mudanças que permeiam as tendências. 
Não obstante, isto não elimina a necessidade de considerar outras práticas de cariz sociológico ou etnográfico, das entrevistas e inquéritos à observação participante (RECH, 2020). Para o propósito deste artigo sublinhamos a imersão urbana e a experiência estética, bem como a ciência por trás da psicogeografia. O trabalho de Careri (2013) apresenta-se como uma perspetiva que muito adiciona à análise de tendências socioculturais neste contexto da cidade. Gomes et al. (2018) e Cantú et al. (2019) sublinharam o conceito de walkscapes de Careri (2013) como uma abordagem capaz de fornecer uma visão sobre as práticas e movimentações urbanas para melhor contextualizar o método do Coolhunting. Não obstante, contribui também para uma visão e observação de largo espectro sobre as dinâmicas urbanas e as relações entre grupos, tribos e indivíduos, numa constante produção e reprodução de "práticas significantes" (HALL, 1997).

O caminhar como ato cognitivo e criativo apresenta-se como uma forma de configurar e reconfigurar o espaço urbano; para além de caracterizar uma forma de arte e uma prática estética, como sugere Careri (2013), o caminhar é uma prática ao mesmo tempo investigativa e criativa. A relação com o espaço urbano é comum à prática artística, obtendo especial atenção das vanguardas artísticas do início do século XX. O caminhar permite desenvolver mapeamentos e cartografias urbanas (O'ROURKE, 2013), considerando tanto os aspectos sensoriais, estéticos e simbólicos da ambiência urbana quanto os transeuntes e os seus modos de vida.

As chamadas práticas de inspiração etnográfica (HUNT, 2011), incluem não somente as etnografias de curta duração e com objetivos específicos realizadas com fins mercadológicos ou projetuais (KNOBLAUCH, 2005; PINK, 2008, 2013), mas também um envolvimento poético, estético e artístico de um modo de pesquisa que considera a fenomenologia do espaço urbano (JENKS e NEVES, 2000; CARERI, 2013; O'ROURKE, 2013; HINDLEY, KNOWLES e RUTH, 2015; ALVES, 2016; RIZK e BIRIOUKOV, 2017). Da flânerie baudelaireana, como ferramenta narrativa (JENKS e NEVES, 2001; BASTOS, 2016; RIZK \& BIRIOUKOV, 2017), às práticas das vanguardas, como a cidade banal do movimento Dadá, o tempo onírico da deambulação surrealista (CARERI, 2013) e as psicogeografias situacionistas, destacam-se as posturas estéticas, simbólicas e investigativas frente ao espaço urbano que delineiam práticas e questões do modo de investigação dos Estudos de Tendências.

\section{Potenciais Aplicações e Matrizes de Investigação}

Em 2016, Gomes propôs uma matriz capaz de articular diferentes modelos/exercícios analíticos que partiam dos Estudos de Tendências ou que se cruzavam com estes. Partiu dos processos de identificação de tendências, da análise de estilos de vida, da análise do espírito do tempo e sublinhou a importância da inovação (GOMES, 2016). Queremos construir em cima desta ideia, mas com um foco nas potenciais articulações metodoló- 
gicas que cruzem tendências socioculturais já identificadas, bem como os seus insights, com os resultados da aplicação dos métodos discutidos. Como já vimos, o processo e os métodos envolvidos na identificação de tendências já estão documentados, pelo que é importante agora usar os seus resultados - as tendências identificadas - em sintonia com métodos escolhidos para solucionar determinados problemas e responder a questões específicas.

- Os estudos de tendências e uma gestão estratégica da cultura. No contexto de uma abordagem de análise cultural orientada para fins estratégicos, existem vários cruzamentos possíveis entre o branding e estratégia culturais (BATEY, 2016; TORELLI, 2013; HOLT e CAMERON, 2010; HEDING et al., 2009; CAYLA e ARNOULD, 2008; O'REILLY, 2005; HOLT, 2004 ) e o marketing tribal (HAWKINS, 2018; CANNIFORD, 2011; COVA e COVA, 2002), conforme mapeado por Gomes (2019). Esta análise cultural com fins estratégicos pode beneficiar da inclusão da leitura de macro e micro tendências socioculturais, acrescentado uma perspetiva macro sobre fenômenos, narrativas e públicos, no âmbito de padrões de consumo e nas ideias criativas que estão na base da desconstrução do mundo simbólico ${ }^{5}$.

- Triangulação cultural. Porta e Keating (2008) contextualizam a triangulação de métodos como uma forma de cruzar perspetivas e fundir diferentes abordagens, que se complementam, numa única que pode ter lugar em diferentes momentos, sublinhando, inclusive, as entrevistas e a análise textual. Martin Raymond (2010) ressaltou a importância da triangulação cultural na análise de tendências. Apesar deste mapeamento, importa ter em consideração a exigência, o contexto e o procedimento específico de cada método, das entrevistas (idealmente semi-estruturadas) até aos inquéritos que o autor indica ${ }^{6}$. Podem ser obtidos tanto dados quantitativos como qualitativos. Estes ligamse à observação participante no local de análise. Uma prática tão complexa como a análise de tendências socioculturais beneficia desta articulação de métodos para compreender tanto a produção de significados como a sua performance, desconstrução e recepção (RECH e NASCIMENTO, 2017). 
- Tendências e Semiótica. Como Silva (2015), Gomes (2020) e Lobo et al. (2021) já abordaram - a primeira ao nível da semiótica americana e de Peirce e o segundo ao nível da semiologia de Saussure e Barthes - é possível articular os resultados das leituras semióticas com o ADN/natureza das tendências. Neste âmbito, ao compreender os significados denotativos e conotativos presentes no objeto de estudo, bem como os potenciais mitos associados, deciframos o mesmo no seu contexto sociocultural. Os resultados das leituras podem ser revistos à luz dos textos descritivos das tendências socioculturais, encontrando paralelos que enquadram o objeto nos principais movimentos socioculturais. Isto potencia um diagnóstico, por exemplo, de peças de comunicação.

- Tendências e o espaço urbano. Da experiência estética à investigação nas ruas. A perspectiva fenomenológica da pesquisa no espaço urbano, bem como as relações estéticas, críticas e interpretativas que sugere, é capaz de prover não apenas dados, mas uma nova forma de pesquisa que considera a imersão do pesquisador no contexto pesquisado. Deste modo, nos Estudos de Tendências, o caminhar não é apenas uma prática artística ou estética, como sugere Careri (2013) nos walkscapes, mas uma nova forma de descrever um modo de pesquisa capaz de prover dados criativos que podem ser analisados a fim de gerar insights a respeito das mudanças de mentalidades e oportunidades emergentes no complexo cenário contemporâneo (CANTÚ et al., 2019)

- O coolhunting, uma prática plural. O coolhunting desde cedo tem estado em foco como prática privilegiada, ou específica, dos Estudos de Tendências que procura identificar e analisar objetos/sinais cool, como podemos ver em Gladwell (1997). Mas dos seus desenvolvimentos profissionais até à tradução processual que diferentes autores (DRAGT, 2018; GOMES et al., 2018; KONGSHOLM e FREDERIKSEN, 2018; ROHDE, 2011) indicam num contexto de "manual" e à definição do próprio conceito de cool (LOBO et al., 2020; GOMES et al., 2018; ROHDE, 2011), esta prática ainda permanece no campo do empírico. No ponto de vista científico, ainda permanece por fechar uma associação metodológica sólida com base num processo científico replicável e com amostras válidas. 
A natureza destes objetos/sinais cool que o coolhunting procura pode também ser articulada, com a devida adaptação conceptual, ao conceito de inovações culturais $^{7}$ proposto por Holt e Cameron (2010). Na nossa perspetiva, o próximo passo nesta reflexão passa por compreender o coolhunting - e especificamente a observação, registo e análise do sinal cool - como uma prática que articula: (1) o método etnográfico desde a observação participante até potencialmente as entrevistas a ter lugar no espaço da observação - e a deriva como contextualizada pelas walkscapes de Careri (2013) - que permite uma exploração do espaço urbano e o registo de elementos sinestésicos. Do ponto de vista do espaço físico, esta é a abordagem de observação e de registo necessária; (2) a prática semiótica, na medida em que cada sinal identificado deve ser desconstruído ao nível dos seus significados num plano denotativo e conotativo. Esta leitura permite compreender o sentido e o impacte do sinal, bem como o seu contexto na produção de significados, ou seja, o que significa e qual é o germe criativo por trás da sua criação; (3) uma dimensão contextual onde o sinal - criativo e/ou cool - habita um espaço que pode não ser cool e ter uma natureza mais sólida. A compreensão do objeto cool face ao estatuto que o rodeia sublinha a dicotomia entre cultura lenta e rápida que McCracken sublinhou (2011) e coloca em destaque a natureza específica do sinal e a sua disrupção face ao contexto que habita e o rodeia.

- Séries de dados - a análise de conteúdo. Por sua vez, a análise de conteúdo facilita a sistematização dos sinais cool/criativos, discutidos no ponto anterior e a sua categorização, mediante categorias temáticas ou atributos físicos/funcionais específicos. Aliás, a análise de conteúdo facilita também a sistematização de dados recolhidos numa pesquisa secundária (desk research), ou mediante a análise sistemática de grandes séries de informação. 


\section{Considerações Finais}

Como vimos, já encontramos ensaios que procuram sistematizar processos e práticas de análise de tendências num contexto de mudanças e padrões de comportamento. No entanto, a classificação e o estado ainda imberbe dos Estudos de Tendências no âmbito dos Estudos de Cultura justifica reflexões que procurem articular quadros conceptuais-metodológicos e métodos específicos com as práticas de identificação e análise de tendências. Este é um exercício de definição epistemológica. Considerando que a pesquisa de tendências é uma atividade de foro tanto etnográfico como hermenêutico, o papel de métodos como a semiótica é claro. A semiótica permite uma leitura em profundidade dos objetos das tendências, enquanto signos, no seu âmbito denotativo e conotativo, seguindo a perspetiva Saussuriana e Barthesiana. Para além disto, o exercício semiótico permite descodificar mitos, construções conotativas complexas, que habitam os sinais e objetos das tendências. Por sua vez, como sublinhado, a análise de conteúdo revela grandes padrões nos dados, em termos de repetições de fenômenos/objetos ou até de categorias. Considerando que estudar tendências passa por sistematizar um largo número de informações, fica clara a relação. Noutro espectro, as práticas de inspiração etnográfica no âmbito de uma imersão urbana coordenam esforços de observação participante e de contato com agentes criativos e públicos. Esta articulação de métodos e práticas baliza as práticas de análise de tendências e confere processos reconhecidos e validados em contexto científico.

1 Este estudo contou com o financiamento da Coordenação de Aperfeiçoamento de Pessoal de Nível Superior - Brasil (CAPES) - Código do financiamento 001

2 Ao mencionar a ideia de 'modo de vida', Powers (2019, pp. 33-35) refere-se diretamente ao conceito de "way of life" de Raymond Williams (1965).

3 Tradução livre do original: "The sign is therefore a compound of signifier and signified. The plane of the signifiers constitutes the plane of expression and that of the signifieds the plane of content" (BARTHES, 1986, p. 39)

4 Tradução livre do original: "The signifiers of connotation, which we shall call connotators, are made up of signs (signifiers and signifieds united) of the denoted system" (BARTHES, 1986, p. 91)

5 Gomes (2019) sublinha que a análise de tendências tem um grande potencial intervir no processo de definição e revisão de expressões culturais no âmbito de uma estratégia cultural (conforme definida por Holt e Cameron, 2010), sublinhando a ligação entre os Estudos de Tendências e uma Gestão Estratégica da Cultura. Por sua vez, Devon Powers indica que o "conhecimento de tendências é vendido como estratégia cultural" (2019, p. 102, tradução nossa), reflectindo sobre o conceito de Chief Culture Officer de McCracken (2010) e sobre a lógica da estratégia cultural de Holt e Cameron (2010) na indústria das tendências (POWERS, 2019, p. 103-106). 
6 Para o autor: "Cultural triangulation is a combination of three distinct but mutually beneficial processes [...]. They are: interrogation: the use of quantitative survey techniques, including household surveys and expert interviews, to poll individuals or groups about their attitudes or activities, with a view to determining the percentage breakdown of these attitudes or activities in relation to the larger population or cultural mainstream; observation: the use of a set of qualitative tools such as ethnography and visual profiling to shadow and observe individuals or consumers involved in a particular task or lifestyle activity, with a view to learning more about that task or activity; intuition: drawing on your experiences as a forecaster to add a further layer of insight to the qualitative and quantitative underpinnings acquired during the interrogation and observation stages" (RAYMOND, 2010, p. 120).

7 No âmbito de uma estratégia cultural e de práticas de gestão de marcas, Holt e Cameron (2010) ilustram a natureza da inovação cultural como a construção de uma expressão cultural inovadora: "The engine of cultural innovation is historical change in society that is significant enough to destabilize the category's cultural orthodoxy, creating latent demand for new cultural expressions [...] at some point, as history unfolds and social structure shift, one or more of these shifts will be disruptive, challenging the taken-for-granted cultural expressions offered by category incumbents, and creating emergent demand for new cultural expressions. This is what we call a social disruption. These are moments when once-dominant brands lose their resonance and when innovative brands take off because they deliver the right expression"(HOLT e CAMERON, 2010, p. 185).

\section{Referências}

BARDIN, Laurence. Análise de Conteúdo. Edições 70: Lisboa, 1979.

BARTHES, Roland. Elements of Semiology. New York: Hill and Young, 1986.

BARTHES, Roland. Mythologies. Traduzido por Annette Lavers. New York: Noonday, 1972.

BASTOS, Paulo Bernardino. À deriva com smartphone. DAT Journal, v. 1, n. 2, p. 134-150, 2016.

BATEY, M. Brand Meaning: Meaning, Myth and Mystique in Today's Brands. London: Routledge, 2016.

CAMPOS, Amanda Queiroz. Taxonomia dos tipos de tendências: uma proposta concisa. DAT Journal, v. 5, n. 2, p. 313-328, 2020.

CANNIFORD, R. A typology of consumption communities. Research in Consumer Behavior, vol. 13, pp. 57-75, 2011.

CANTÚ, William; LOPES, Clarissa A.; GOMES, Nelson P.; PRADO, Gilbertto. The Gaze of Culture and Communication: Strategic Analysis of Consumption and Trends. In E. Duarte (Ed.) Design Doctoral Conference'19: TRANSformation. Proceedings of the DDC 6th Conference, Lisboa: Edições IADE, 2019.

CARERI, F. Walkscapes: o caminhar como prática estética. São Paulo: Gustavo Gili, 2013. CAYLA, J.; ARNOULD, E. J. A Cultural Approach to Branding in the Global Marketplace. Journal of International Marketing, vol. 16, nํ. 4, p. 88-114, 2008. 
COVA, Bernard; COVA, Veronique. Tribal Marketing: The tribalization of society and its impact on the conduct of marketing. European Journal of Marketing, special issue on Societal Marketing in 2002 and Beyond, 2002.

CRAMER, Tessa; DUIN, Patrick Van Der; HESELMANS, C. Trend Analysis. In.: Patrick van der Duin (ed), Foresight in Organizations - Methods and Tools. NY: Routledge, 2018.

DRAGT, Els. How to Research Trends. Amsterdam: BIS Publishers, 2017.

GLADWELL, M. The coolhunt: who decides what's cool? Certain kids in certain places-and only the coolhunters know who they are. The New Yorker, 1997.

GOMES, Nelson P. A Análise Cultural e o Estudo de Tendências na Comunicação e Gestão de Marcas: Estudo de caso publicitário em contexto semiótico. International Journal of Marketing, Communication and New Media, Special Issue 7 - QRMCNM, pp. 56-79, 2020.

GOMES, Nelson P. A análise de Tendências e da Cultura como uma ferramenta para a Gestão de Marcas. e-Revista Logo, vol.4, nº 1, pp. 59-72, 2015.

GOMES, Nelson P. The Management of Culture: Professional Challenges of Managing Narratives and Brands in a changing Cultural Environment. e-Revista Logo, vol.6, no1, 2017.

GOMES, Nelson P. Trends Management applied to Branding and Cultural Management. e-Revista Logo, vol. 5, ํํ. 1, 2016.

GOMES, Nelson, COHEN, Suzana; FLORES, Ana Marta. Estudos de Tendências: Contributo para uma abordagem de análise e gestão da Cultura. Moda Palavra, vol. 11, ํo. 22, 2018.

GOMES, Nelson. Gestão da cultura: estratégia e articulações no âmbito do branding e das tribos urbanas. E-revista LOGO, vol. 8, nº. 2, 2019.

HALL, Stuart (Ed.). Representation: Cultural Representations and Signifying Practices. Walton Hall: The Open University, 1997.

HAWKINS, M. A. Researching and marketing to consumption collectives. International Journal of Market Research, 2018.

HEDING, T.; KNUDTZEN, C.F.; BJERRE, M. Brand Management: Research, theory and Practice. New York: Routledge, 2009.

HIGHAM, William. The Next Big Thing. London: Kogan Page, 2009.

HOLT, Douglas; CAMERON, Douglas. Cultural Strategy: using innovative ideologies to build breakthrough brands. Oxford: Oxford University Press, 2010.

HOLT, Douglas. How Brands Become Icons: The Principles of Cultural Branding. Boston: Harvard Business School Press, 2004.

INGLIS, Fred. Cultural Studies. Cambridge: Blackwell, 1993.

KONGSHOLM, L.; FREDERIKSEN, C. Trend Sociology v. 2.0. Herning: Pej Gruppen, 2018.

LOBO, Theresa; CANTÚ, William A.; GOMES, Nelson P. Cork as a Relevant Material in Fashion: A Study of Socio-cultural Trends and a Semiotic Reading of the Cork-a-Tex Yarn. In W. Karwowski et al. (eds.) Human Systems Engineering and Design III: Future Trends and Applications, Switzerland: Springer, 2021.

MARGOLIN, Victor. Design, the future and the human spirit. Design Issues, v. 23, n. 3, p. 4-15, 2007.

MASON, Henry; MATTIN, D.; LUTHY, M.; DUMITRESCU, D. Trend Driven Innovation. New Jersey: Wiley, 2015.

MCCRACKEN, Grant. Chief Culture Officer - How to create a living, breathing corporation. New York: Basic Books, 2011 [2009]. 
O’REILLY, D. Cultural Brands/Branding Culture. Journal of Marketing Management, n²1, 2005. OSWALD, Laura. Marketing Semiotics: signs, strategies and brand value. UK: Oxford University Press, 2012.

PORTA, D.; KEATING, M. Approaches and Methodologies in the Social Sciences: A pluralist Perspective. Cambridge: Cambridge University Press, 2008.

Powers, D. (2018). Thinking in trends: the rise of trend forecasting in the United States. Journal of Historical Research in Marketing. n. 1, v. 10, pp. 2-20.

POWERS, Devon. Trend - The business of Forecasting the Future. Chicago: University of Illinois Press, 2019.

PRADO, Gilbertto; TAVARES, Monica; ARANTES, Priscila (Org.). Diálogos transdisciplinares: arte e pesquisa. São Paulo: ECA/USP, 2016.

RAYMOND, Martin. Trend Forecaster's Handbook. London: Laurence King, 2010.

RECH, Sandra. (2020). Trends Management: The Qualitative Approach as a Methodology. In Raposo D., Neves J., Silva J. (Ed.), Perspective on Design. Springer Series in Design and Innovation, vol 1.

RECH, Sandra. Contributo da Pesquisa Qualitativa para a Consolidação Disciplinar dos Estudos de Tendências: processo, perspectivas e corpus. Convergências, vol. X, ㄲo․ 19, 2017.

RECH, Sandra.; CAPONERA, Letícia. (2018). O trendspotting e sua importância nos Estudos de Tendências. $14^{\circ}$. Colóquio de Moda. Paraná: PUCPR.

RECH, Sandra.; NASCIMENTO, Janaina. (2017). Triangulação nos Estudos de Tendências. 13². Colóquio de Moda. São Paulo: UNESP.

RECH, Sandra.; SEIBEL, S.; FELIPPE, A. Trend Research and Artificial Intelligence: the case of the company Coleção.Moda. E-Revista LOGO, vol. 9, no. 1, 2020.

RECH, Sandra.; SILVEIRA, Icléia. (2017). Abordagem Terminológica dos Estudos de Tendências. 13 ํㅡó Cóquio de Moda. São Paulo: UNESP.

ROGERS, E. M. Diffusion of Innovations. New York: Free Press, 2003 [1962].

ROHDE, Carl. Serious Trendwatching. Tilburg: Fontys University of Applied Sciences and Science of the Time, 2011.

ROSE, Gillian. Visual Methodologies: In Introduction to the Interpretation of Visual Materials. London: SAGE Publications, 2001.

SANTAELLA, Lucia. Estética da Fascinação. DAT Journal, v. 4, n. 3, p. 22-36, 6 dez. 2019.

SANTOS E SILVA, Janiene. Tendências Socioculturais: Recorrências Simbólicas do Espírito do Tempo no Sistema Publicitário. Tese de Doutoramento em Ciências da Comunicação. São Paulo: Universidade de São Paulo, 2015.

TORELLI, C. J. Globalization, Culture, and Branding: How to Leverage Cultural Equity for Building Iconic Brands in the Era of Globalization. New York: Palgrave Macmillan, 2013. VEJLGAARD, Henrik. Anatomy of a Trend. New York: McGraw-Hill, 2008.

VISONÁ, Paula. Sobre o Estar-Junto-Com Como Tendência Sociocultural e Coletivos Criativos: uma proposta interdisciplinar de metodologia para identificação de tendências visando cenários de futuro. Moda Palavra,vol. 11, №. 22, 2019.

ZUANON, Rachel; PRADO, Gilbertto; FERREIRA, Claudio. Transdisciplinaridades: Projetos, materiais e processos. DAT Journal, v. 1, n. 2, p. 1, 27 dez. 2016.

WILLIAMS, Raymond. The Long Revolution. London: Penguin Books, 1965 [1961].

WIMER, R.; DOMINICK, J. Mass Media Research. Boston: Wadsworth, 2011.

Recebido: 06 de janeiro de 2021. Aprovado: 10 de fevereiro de 2021. 